\title{
Maximal Triangle Free Graph and Traveling Salesman Problem
}

\author{
Jayanta $\mathrm{Kr}$ Choudhury \\ Swadeshi Academy Junior College, \\ Guwahati-781005, India
}

\author{
Bichitra Kalita \\ Department of M.C.A.(Computer Application), \\ Assam Eng. College, Guwahati-781013, India
}

\begin{abstract}
In this paper, a maximal triangle free graph has been generated from the complete graph $K_{2 m+3}$ for $m \geq 2$ by deleting $\left(m^{2}+3 m+1\right)$ number of edges. In addition, two theorems have been established for it. Finally, an algorithm has been developed under different cases to solve the traveling salesman problem when the weights of the edges are non-repeated of the complete graph $K_{2 m+3}$ for $m \geq 2$.
\end{abstract}

\section{Keywords}

Algorithm, Maximal Triangle Free Graph (MTFG), Complete Graph, Hamiltonian Graphs, Traveling Salesman Problem (TSP).

\section{INTRODUCTION}

One of the areas of Mathematics which is most commonly used in all fields of scientific investigation is that of graph theory. Graph theory has wide range of applications in computer science, networking problems and in connectivity. The triangle free graph is an undirected graph in which no three vertices form a triangle of edges, and it has many properties. The triangle finding problem is the problem of determining whether a graph is triangle free or not. When the graph does contain a triangle, algorithms are often required to output three vertices which form a triangle in the graph. One way to generate triangle free graphs in which all independent sets are small is the triangle free process in which one generates a maximal triangle free graph by repeatedly adding randomly chosen edges that do not complete a triangle. Much research about triangle free graph has been focused on graph theory. Researcher like Woodall[1], Shi[2], Goddard[3], Furedi[4], Briegmann[5] and Brandt[6] produced important work on triangle free graph. In 1973, Woodall defined the binding number of a graph, $\operatorname{bind}(G)$, and obtained some useful results and conjectures. It is known that the binding number of a $\operatorname{graph}(G)$, $\operatorname{bind}(G)$, is the largest number $c$ such that $|N(X)| \geq \min (c|X|,|V(G)|)$ for every sub set $X \subseteq V(G)$, where $V(G)$ is the vertex set of $G$, and $N(X)=\cup_{u \in X} N(u), N(u)$ denotes the set of vertices adjacent to $u$. He further proposed some conjectures related to binding number which reflected the property of Hamiltonian graph. Shi[2], in his paper, proved the Woodall's conjecture B[1], "if $\operatorname{bind}(G) \geq \frac{3}{2}$, then $G$ contains a triangle. Shi[2] also proved that if $\operatorname{bind}(G) \geq \frac{3}{2}$, then each vertex is contained in a 4-cycle, each edge is contained in a 5-cycle when $V(G) \geq 11$ and there exists a 6-cycle in $G$. Goddard and Kleitman[3] shown that a minimal triangle free graph on $n$ vertices with minimum degree $\delta$ contains an independent set of size $(3 \delta-n)$ vertices which have identical neighborhoods. They also proved Woodall's conjecture $\mathrm{B}[1]$ that if the binding number of a graph is at least $\frac{3}{2}$, then it has a triangle and they claim that their proof is much simpler than Shi's[2] proof of the triangle part of the Woodall's conjecture B[1]. Füredi, Reimer \& Seress[4] in their paper established some useful results for maximal triangle free graph. András Hajnal proposed the triangle free game. Starting with the empty graph on $n$ points, two players alternatingly pick edges with restriction that no player may complete a triangle. The score is the total number of edges drawn and the first player's objective is to obtain as high as possible. Füredi, Reimer \& Seress[4] showed that the first player can achieve a score of $\Omega(n \log n)$. The result will follow from a lower bound on the minimum number of edges in a maximal triangle free graph containing a large matching. More generally, they determine the asymptotic behavior of the minimum number of edges in maximal triangle free graphs containing a matching of size $\lfloor n / 2\rfloor$ and having each vertex valency $\leq D$. Brügman, Komusiewicz and Moser[5] shown that the problem to decide whether a graph can be made triangle free with at most $k$ edge deletions remains NP-complete even when restricted to a planar graph of maximum degree seven. Moreover, Brügman, Komusiewicz and Moser[5], provide polynomial-time data reduction rules for this problem and obtain problem kernels consisting of $6 k$ vertices for general graph and $\frac{11 k}{3}$ vertices for planar graphs. Brandt, Brinkmann and Harmuth[6], present an efficient algorithm for generating maximal triangle free graph. A program based on this algorithm has been used to check a conjecture of Erdös about the local density of triangle free graphs and turned out to be very powerful for the computation of triangle Ramsey numbers. But the properties of triangle free graph for traveling salesman problem has been rarely used. It is well known that the traveling salesman problem is a NP-complete problem and there is no algorithm to find out the least cost route of a traveler for any arbitrary Hamiltonian graph. Kalita[7] forwarded some methods to determine the least cost route of a traveler of the complete weighted graph. Thereafter Kalita[8] developed another technique to determine the least cost route of traveling salesman problem by applying the TEEP graphs. Moreover, Kalita[9] discussed some structures of non-isomorphic Hamiltonian sub graphs of complete graph and forwarded a new technique to determine the least cost route of traveling salesman problem. Recently, Kalita[10] studied some structures of simple non-isomorphic Hamiltonian sub graphs of the form $H(2 m+3,6 m+3)$ in the complete graph $K_{2 m+3}$ for $m \geq 2$. Further he found various structures of the form $H(2 m+3,6 m+3)$ for $m \geq 2$ and some of them have been found to give some forms of metal atom cluster compound of chemistry. Finally, Kalita[10] established an algorithm to solve the traveling salesman problem when the weights of edges of the complete graph $K_{2 m+3}$ for $m \geq 2$ are non-repeated. Dutta, Kalita and Baruah[11] studied Hamiltonian circuits and edge disjoint Hamiltonian circuits of various types of regular sub graph of the complete graph $K_{2 m+2}$ for $m \geq 2$. They also discussed the perfect matching of this type of regular sub graph of various degrees and finally, they have forwarded an algorithm to solve the traveling salesman problem for different cases. Recently, they discussed in the paper[12] about the application of regular planar sub graphs of the complete graph $K_{2 m+2}$ for $m \geq 2$. In addition, they have developed an algorithm to determine the best possible shortest route of traveling salesman problem. Very recently, a heuristic 
technique for traveling salesman problem has been discussed in [13] under different cases of the complete graph $K_{2 m+2}$ for $m \geq 2$, i.e., when $2 m+2$ consecutive greatest weights for $m \geq 2$ are incident with a vertex and $2 \leq$ number of equal weights $\leq 2 m$ are incident with another vertex. In addition, Choudhury and Kalita[13] forwarded two theorems. Choudhury, Dutta and Kalita[14] study different types of factorial graphs of the complete graphs $K_{6 m-2}, K_{6 m+2}$, $K_{6 m}$ for $m \geq 1$. Some theoretical investigation related to 3 -factors, 2-factors and 1-factors have been discussed in [14]. Finally, an algorithm for traveling salesman problem has been developed.

In this paper, a theorem has been established to construct a maximal triangle free graph from the complete graph $K_{2 m+3}$ for $m \geq 2$ and thereafter, a theorem is forwarded to find the number of Hamiltonian circuits in the maximal triangle free graph. Finally, an algorithm has been developed to determine the least cost route of a traveler.

The paper is organized as follows: The section 1 includes the introduction part containing the works of other researchers. Section 2 includes notations and terminologies. In section 3 , two theorems are stated and proved. Section 4 includes an algorithm. Section 5 explains experimental result, and the conclusion is included in section 6 .

\section{NOTATION AND TERMINOLOGY}

The notation and terminologies are drawn from the standard references [1-14]. The complete graph $K_{2 m+3}$ for $m \geq 2$ is considered to generate a maximal triangle free graph. It is known that a maximal triangle free graph is one which does not contain the triangle $K_{3}$ but the addition of any edge would create a triangle. The cost matrix of a traveler is always considered for odd number cities.

Consider the complete graph $K_{2 m+3}$ for $m \geq 2$. There are $(2 m+3)$ vertices and $(2 m+3)(m+1)=2 m^{2}+5 m+3$ edges in the complete graph $K_{2 m+3}$ for $m \geq 2$, the degree of each of the vertex being $(2 m+2)$. It is well known that the number of Hamiltonian circuits in $K_{2 m+3}$ for $m \geq 2$ is $\frac{(2 m+2) !}{2}$.

\section{THEOREMS}

3.1 Theorem: Let $K_{2 m+3}$ for $m \geq 2$ be a complete graph. If $\left(m^{2}+3 m+1\right)$ number of edges are deleted from the complete graph $K_{2 m+3}$ in such a way that the degree of one vertex is always two and the degree of remaining $(2 m+2)$ number of vertices is $(m+1)$, then there exists a maximal triangle free graph.

Proof: The theorem will be proved for three cases.

Case 1: When all edges of the cycle $C_{2 m+3}$ of the complete graph $K_{2 m+3}$ for $m \geq 2$ are fixed.

Proof: Let $K_{2 m+3}$ for $m \geq 2$ be a complete graph. Keeping all edges of the cycle $C_{2 m+3}$ for $m \geq 2$ fixed, let $(2 m+2)$ number of edges connecting the alternate vertices i.e., $\left\{v_{1} v_{3}, v_{2} v_{4}, v_{3} v_{5}, v_{4} v_{6}, \cdots \cdots, v_{2 t-1} v_{2 t+1}, v_{2 s} v_{2 s+2}, v_{2 m+3} v_{2}\right\}$ where $1 \leq t \leq m+1,1 \leq s \leq m$ for $m \geq 2$ be deleted. Then the complete graph $K_{2 m+3}$ for $m \geq 2$ reduces to a graph in which degree of each of the $(2 m+1)$ vertices become $2 m$ and other two vertices viz. $v_{1}$ and $v_{2 m+2}$ having degree $(2 m+1)$ for $m \geq 2$ [Figure-2 for $m=2$ and Figure-6 for $m=3$ ]. Now arranging the $(2 m+2)$ number of deleted edges as mentioned above in successive order as

$$
\left\{\begin{array}{c}
v_{1} v_{3}, v_{3} v_{5}, v_{5} v_{7}, \cdots, v_{2 t-1} v_{2 t+1}, v_{2 m+3} v_{2}, v_{2} v_{4}, v_{6} v_{8}, \cdots, \\
v_{2 s} v_{2 s+2}
\end{array}\right\}
$$

where $1 \leq t \leq m+1,1 \leq s \leq m$ for $m \geq 2$ it is found that they form a Hamiltonian path. Let this path be denoted by $P$. Then

$$
P=v_{1} v_{3} v_{5} \cdots \cdots v_{2 t-1} v_{2} v_{4} v_{6} \cdots \cdots v_{2 s}
$$

where $1 \leq t \leq m+2,1 \leq s \leq m+1$ for $m \geq 2$. Let the edge $v_{2 m+2} v_{1}$ for $m \geq 2$, joining the two vertices $v_{1} \& v_{2 m+2}$ having degree $(2 m+1)$ be deleted. Then the complete graph $K_{2 m+3}$ for $m \geq 2$ reduce to a regular graph of degree $2 m$ for $m \geq 2$ [Figure-3 for $m=2$ and Figure-7 for $m=3$ ]. Now adding the edge $v_{2 m+2} v_{1}$ for $m \geq 2$ with the path $P$, a Hamiltonian circuit is formed, which contains all $(2 m+2)$ number of earlier deleted edges for $m \geq 2$. Let the Hamiltonian circuit be denoted by $W$. Then

$$
\begin{aligned}
& \qquad W=v_{1} v_{3} v_{5} \cdots \cdots v_{2 t-1} v_{2} v_{4} v_{6} \cdots \cdots v_{2 s} \\
& \text { where } 1 \leq t \leq m+2,1 \leq s \leq m+1 \text { for } m \geq 2
\end{aligned}
$$

After deletion of the Hamiltonian circuit $W$, containing $(2 m+3)$ number of edges from the complete graph $K_{2 m+3}$ for $m \geq 2$, another $\left(m^{2}+m-2\right)$ number of edges are deleted keeping in mind that all edges of the cycle $C_{2 m+3}$ for $m \geq 2$ remain fixed. These $\left(m^{2}+m-2\right)$ numbers of edges to be deleted in the following way (Table-1) for different values of $m$.

After deleting altogether $\left(m^{2}+3 m+1\right)$ number of edges, the complete graph $K_{2 m+3}$, for $m \geq 2$ reduces to a maximal triangle free graph. This maximal triangle free graph contains $\left(m^{2}+2 m+2\right)$ number of edges in which one vertex has degree two and the degree of each of the remaining $(2 m+2)$ vertices is $(m+1)$ [Figure-4 for $m=2$ and Figure- 8 for $m=2]$. This completes the proof.

Case2: When all edges of the cycle $C_{2 m+3}$ of the complete graph $K_{2 m+3}$ for $m \geq 2$ are deleted.

Proof: Let $K_{2 m+3}$ for $m \geq 2$ be a complete graph. Let $(2 m+2)$ number of edges incident at different vertices along the cycle $C_{2 m+3}$ be deleted. Let these $(2 m+2)$ number of edges be $\left\{v_{1} v_{2}, v_{2} v_{3}, \cdots \cdots, v_{t} v_{t+1}\right\}$ where $1 \leq t \leq 2 m+2$ for $m \geq 2$. Then the complete graph $K_{2 m+3}$ for $m \geq 2$ reduces to a graph in which degree of each of the $(2 m+1)$ vertices becomes $2 m$ and two vertices viz. $v_{1}$ and $v_{2 m+3}$ having degree $(2 m+1)$ for $m \geq 2$ [Figure-10 for $m=2$ and Figure-14 for $m=3]$. Now these $(2 m+2)$ numbers of deleted edges form a Hamiltonian path. Let this path be denoted by $Q$. That is $Q=v_{1} v_{2} v_{3} v_{4} \cdots \cdots v_{t} v_{t+1}$ where $1 \leq t \leq 2 m+2$ for $m \geq 2$. Let the edge $v_{2 m+3} v_{1}$ joining the two vertices $v_{1}$ and $v_{2 m+3}$ for $m \geq 2$ having degree $(2 m+1)$ be deleted. Then the complete graph $K_{2 m+3}$ for $m \geq 2$ reduce to a regular graph of degree $2 m$ for $m \geq 2$ [Figure- 11 for $m=2$ and Figure- 15 for $m=3$ ]. Now adding the edge $v_{2 m+3} v_{1}$ for $m \geq 2$ with path $Q$, a Hamiltonian circuit is formed which contains all $(2 m+2)$ number of earlier deleted edges for $m \geq 2$. Let the Hamiltonian circuit be denoted by $T$.

Then $\quad T=v_{1} v_{2} v_{3} v_{4} v_{5} v_{6} v_{7} \cdots \cdots v_{t} v_{t+1} v_{2 m+3} v_{1} \quad$ where $1 \leq t \leq 2 m+2$ for $m \geq 2$.

After deletion of the Hamiltonian circuit $T$, containing $(2 m+3)$ number of edges from the complete graph $K_{2 m+3}$ for $m \geq 2$, another $\left(m^{2}+m-2\right)$ number of edges for $m \geq 2$ are deleted. These $\left(m^{2}+m-2\right)$ number of edges for $m \geq 2$ to be deleted in the following way (Table-2) for different values of $m$.

After deleting altogether $\left(m^{2}+3 m+1\right)$ number of edges, the complete graph $K_{2 m+3}$ for $m \geq 2$ reduces to a maximal triangle free graph. This maximal triangle free graph contains $\left(m^{2}+2 m+2\right)$ number of edges in which one vertex has 
degree two and the degree of each of the remaining $(2 m+2)$ number of vertices is $(m+1)$ [Figure-12 for $m=2$ and Figure-16 for $m=3$ ]. This completes the proof.

Case 3: When $(2 m+2)$ number of edges incident at different vertices of which $(2 m-1)$ number of edges along the cycle $C_{2 m+3}$ of the complete graph $K_{2 m+3}$ for $m \geq 2$ are deleted.

Proof: Let $K_{2 m+3}$ for $m \geq 2$ be a complete graph. Let $(2 m+2)$ number of edges incident at different vertices but few of them, i.e., $(2 m-1)$ number of edges are along the cycle $C_{2 m+3}$ be deleted. Let these $(2 m+2)$ number of edges be

$\left\{v_{1} v_{3}, v_{3} v_{2}, v_{2} v_{4}, v_{t+3} v_{t+4} \cdots \cdots, v_{2 m+1} v_{2 m+3}, v_{2 m+3} v_{2 m+2}\right\}$ where $1 \leq t \leq 2 m-3$ for $m \geq 2$. Then the complete graph $K_{2 m+3}$ for $m \geq 2$ reduces to a graph in which degree of each of the $(2 m+1)$ vertices becomes $2 m$ and two vertices viz. $v_{1}$ and $v_{2 m+2}$ having degree $(2 m+1)$ for $m \geq 2$ [Figure-18 for $m=2$ and Figure-22 for $m=3$ ].

Now these $(2 m+2)$ numbers of deleted edges form a Hamiltonian path. Let this path be denoted by $L$. Then $L=v_{1} v_{3} v_{2} v_{4} \cdots \cdots v_{t+3} v_{t+4} \cdots \cdots v_{2 m+1} v_{2 m+3} v_{2 m+2} \quad$ where $1 \leq t \leq 2 m-3$ for $m \geq 2$. Let the edge $v_{2 m+2} v_{1}$ for $m \geq 2$ joining the two vertices $v_{1}$ and $v_{2 m+2}$ having degree $(2 m+1)$ be deleted. Then the complete graph $K_{2 m+3}$ for $m \geq 2$ reduce to a regular graph of degree $2 m$ for $m \geq 2$ [Figure-19 for $m=2$ and Figure-23 for $m=3$ ]. Now adding the edge $v_{2 m+2} v_{1}$ for $m \geq 2$ with path $L$, a Hamiltonian circuit is formed which contains all the $(2 m+2)$ number of earlier deleted edges for $m \geq 2$. Let the Hamiltonian circuit be denoted by $J$. That is

$J=v_{1} v_{3} v_{2} v_{4} \cdots \cdots v_{t+3} v_{t+4} \cdots \cdots v_{2 m+1} v_{2 m+3} v_{2 m+2} v_{1}$ where $1 \leq t \leq 2 m-3$ for $m \geq 2$.

After deletion of the Hamiltonian circuit $J$, containing $(2 m+$ 3 ) number of edges from the complete graph $K_{2 m+3}$ for $m \geq 2$, another $\left(m^{2}+m-2\right)$ number of edges for $m \geq 2$ are deleted. These $\left(m^{2}+m-2\right)$ number of edges for $m \geq 2$ to be deleted in the following way (Table-3) for different values of $m$.

After deleting altogether $\left(m^{2}+3 m+1\right)$ number of edges, the complete graph $K_{2 m+3}$ for $m \geq 2$ reduces to a maximal triangle free graph. This maximal triangle free graph contains $\left(m^{2}+2 m+2\right)$ number of edges in which one vertex has degree two and the degree of each of the remaining $(2 m+2)$ number of vertices is $(m+1)$ [Figure-20 for $m=2$ and Figure-24 for $m=3$ ]. This completes the proof.

Hence, from the above three cases, it is seen that there exist a maximal triangle free graph.

Theorem 4.3.2: The number of Hamiltonian circuits of the maximal triangle free graph obtained in Theorem 3.1 is always $\{(n+1) !\}^{2}$ for $n \geq 1$ for simultaneous change of $m \geq 2$.

Proof: It is known that the number of edges of the complete graph $K_{2 m+3}$ for $m \geq 2$ is $\left(2 m^{2}+5 m+3\right)$ and the number of Hamiltonian circuits is $\frac{(2 m+2) !}{2}$. It is clear that the existence of the maximal triangle free graph from the complete graph $K_{2 m+3}$ for $m \geq 2$ is possible (Theorem 3.1). It can be shown that there are $\{(n+1) !\}^{2}$ number of Hamiltonian circuits for $n \geq 1$ with simultaneous changes for $m \geq 2$.

It will be proved by induction method. It is seen that the result is true for $n=1$ when $m=2$, i.e., there are $(2 !)^{2}=4$ Hamiltonian circuits when $n=1$ and $m=2$ [Figure-25a to Figure-25d for Case1, as stated in Theorem 3.1 and Figure-25e to Figure-25h for Case $2 \&$ Case 3, as mentioned in Theorem 3.1]. Similarly it can be verified that the number of
Hamiltonian circuits of the maximal triangle free graph obtained from the complete graph $K_{2 m+3}$ is $(3 !)^{2}=6^{2}=36$ when $n=2$ and $m=3$ [only four circuits from Figure-26a to Figure-26d for Case1 and Figure-26e to Figure-26h for Case 2 $\&$ Case 3 is shown].

Suppose that the result is true for $m=k$ and $n=x<k$. Then the number of Hamiltonian circuits is $\{(x+1) !\}^{2}$. Now, to show that the result is true for $m=k+1$ and $n=x+1<k+1$. Since according to the theorem the values of $m$ start from 2, simultaneously, the values of $n$ also start from 1 . Therefore, $k+1 \geq 2 \Rightarrow k \geq 1$ and

$$
\begin{aligned}
{[\{(x+1)+1\} !]^{2} \geq 4 } & \Rightarrow\{(x+2) !\}^{2} \geq(2 !)^{2} \\
& \Rightarrow(x+2) ! \geq 2 ! . \text { This gives } x \geq 0 .
\end{aligned}
$$

$n \geq 1$.

\section{ALGORITHM}

Input: Let $K_{2 m+3}$ for $m \geq 2$ be a complete weighted graph. Output: Find the least cost route.

Case1: When $(2 m+2)$ number of consecutive greatest weights incident at different vertices of the complete graph $K_{2 m+3}$ for $m \geq 2$, but not along edges of the cycle $C_{2 m+3}$.

Step 1: Keeping edges of the cycle $C_{2 m+3}$ for $m \geq 2$ fixed, delete $(2 m+2)$ number of consecutive greatest weights incident at different vertices of the complete graph $K_{2 m+3}$ for $m \geq 2$ such that they form a path.

Step 2: Observe that the degrees of two vertices of the graph obtained in Step 1 become $(2 m+1)$ and the remaining $(2 m+1)$ vertices become $2 m$ for $m \geq 2$.

Step 3: Delete the edge (irrespective of the weight) joining the two vertices having degree $(2 m+1)$ from the graph obtained in Step 2.

Step 4: Observe that the graph obtained in Step 3 is a regular sub graph of the complete graph $K_{2 m+3}$ of degree $2 m$ for $m \geq 2$.

Step 5: From the graph obtained in Step 4, keeping edges of the cycle $C_{2 m+3}$ for $m \geq 2$ fixed, delete another $\left(m^{2}+m-2\right)$ number of edges attached with greatest weights, other than $(2 m+2)$ number of consecutive greatest weights already deleted in Step 1.

Step 6: Observe that the graph $K_{2 m+3}$ for $m \geq 2$ obtained in Step 5 is reduced to a maximal triangle free graph in which degree of one vertex is always two and the degree of each of the remaining $(2 m+2)$ number of vertices is $(m+1)$.

Step 7: Choose the vertex having degree two as the initial vertex from the graph obtained in Step 6.

Step 8: Select the next vertex through the minimum weighted edge.

Step 9: Continue this process of selecting new vertices to the preceding stage of a Hamiltonian circuit.

Step 10: Go to the initial vertex to obtain the Hamiltonian circuit, which will be the least cost route of the traveler.

Step 11: Stop

Case 2: When $(2 m+2)$ number of consecutive greatest weights incident at different vertices along edges of the cycle $C_{2 m+3}$ of the complete graph $K_{2 m+3}$ for $m \geq 2$. Step 12: Delete $(2 m+2)$ number of consecutive greatest weights incident at different vertices along edges of the cycle of the complete graph $K_{2 m+3}$ for $m \geq 2$.

Step 13: Observe that the degrees of two vertices of the graph obtained in Step 12 become $(2 m+1)$ and the remaining $(2 m+1)$ vertices become $2 m$ for $m \geq 2$.

Step 14: Delete the edge (irrespective of the weight) joining the two vertices having degree $(2 m+1)$ from the graph obtained in Step 13. 
Step 15: Observe that the graph obtained in Step 14 is a regular sub graph of the complete graph $K_{2 m+3}$ of degree $2 m$ for $m \geq 2$.

Step 16: From the graph obtained in Step 15, delete another $\left(m^{2}+m-2\right)$ number of edges attached with greatest weights, other than $(2 m+2)$ number of consecutive greatest weights already deleted in Step 12 .

Step 17: Observe that the graph $K_{2 m+3}$ for $m \geq 2$ obtained in step 16 is reduced to a maximal triangle free graph in which degree of one vertex is always two and the degree of each of the remaining $(2 m+2)$ number of vertices is $(m+1)$.

Step 18: Go to step 7 to step 11 .

Case 3: When $(2 m+2)$ number of consecutive greatest weights incident at different vertices but few of them along edges of the cycle $C_{2 m+3}$ of the complete graph $K_{2 m+3}$ for $m \geq 2$.

Step 19: Delete $(2 m+2)$ number of consecutive greatest weights incident at different vertices in which few of them along edges of the cycle of the complete graph $K_{2 m+3}$ for $m \geq 2$.

Step 20: Observe that the degrees of two vertices of the graph obtained in Step 19 become $(2 m+1)$ and the remaining $(2 m+1)$ vertices become $2 m$ for $m \geq 2$.

Step 21: Delete the edge (irrespective of the weight) joining the two vertices having degree $(2 m+1)$ from the graph obtained in Step 20.

Step 22: Observe that the graph obtained in Step 21 is a regular sub graph of the complete graph $K_{2 m+3}$ of degree $2 m$ for $m \geq 2$.

Step 23: From the graph obtained in Step 22, delete another $\left(m^{2}+m-2\right)$ number of edges attached with greatest weights, other than $(2 m+2)$ number of consecutive greatest weights already deleted in Step 19.

Step 24: Observe that the graph $K_{2 m+3}$ for $m \geq 2$ obtained in step 23 is reduced to a maximal triangle free graph in which degree of one vertex is always two and the degree of each of the remaining $(2 m+2)$ number of vertices is $(m+1)$.

Step 25: Go to step 7 to step 11.

\section{EXPERIMENTAL RESULT FOR ALGORITHM}

Example [case1]: The following cost matrix (Table-4) is considered for seven cities (i.e., for $m=2$ of $K_{2 m+3}$ ). From the Table-4, a complete graph of seven vertices is drawn [Figure-27], where the edges of weights $60,56,51,47,42,38$ are incident at different vertices. Now, Step 2 of the algorithm has been applied and the graph obtained is shown in Figure-28. Thereafter, Step 4 as discussed in the algorithm has been applied and the graph so obtained is shown in Figure-29, which is a regular graph. Now, Step 6 of the algorithm has been applied and a maximal triangle free graph is obtained having one vertex of degree two and remaining six vertices of degree three[Figure-30]. Then, Step 7 to Step 12 has been applied to obtain the least cost route as $A \rightarrow B \rightarrow C \rightarrow F \rightarrow E \rightarrow D \rightarrow G \rightarrow A$ with weights equal to 81 . This is the least cost route out of the total Hamiltonian circuit 360 , as $K_{2 m+3}$ has 360 Hamiltonian circuits for $m=2$.

Example [case 2]: The following cost matrix (Table-5) is considered for seven cities (i.e., for $m=2$ of $K_{2 m+3}$ ). From the Table-5, a complete graph of seven vertices is drawn [Figure-31]. Now, Step 13 of the algorithm has been applied and the graph obtained is shown in Figure-32. Thereafter, Step 15 as discussed in the algorithm has been applied and the graph so obtained is shown in Figure-33, which is a regular graph. Now, Step 17 of the algorithm has been applied and a maximal triangle free graph is obtained having one vertex of degree two and remaining six vertices of degree three [Figure-34]. Then, Step 19 to Step 20 has been applied to obtain the least cost route as $A \rightarrow D \rightarrow F \rightarrow B \rightarrow G \rightarrow C \rightarrow E \rightarrow A$ with weights equal to 80 .

Example [case 3]: The following cost matrix (Table-6) is considered for seven cities (i.e., for $m=2$ of $K_{2 m+3}$ ). From the Table-6, a complete graph of seven vertices is drawn [Figure-35]. Now, Step 21 of the algorithm has been applied and the graph obtained is shown in Figure-36. Thereafter, Step 23 as discussed in the algorithm has been applied and the graph so obtained is shown in Figure-37, which is a regular graph. Now, Step 25 of the algorithm has been applied and a maximal triangle free graph is obtained having one vertex of degree two and remaining six vertices of degree three [Figure-38]. Then, Step 27 to Step 28 has been applied to obtain the least cost route as $A \rightarrow E \rightarrow B \rightarrow G \rightarrow C \rightarrow F \rightarrow D \rightarrow A$ with weights equal to 59 is found.

\section{CONCLUSION}

The increases in the number of vertices in a graph definitely increase the complexity of studying some graphs of practical uses which is of academic interest. This demands to reduce the number of vertices and triangle free graph by means of deletion of edges can be helpful in this sense. It is well known to all, that a complete graph of vertices seven consists of 360 Hamiltonian circuits. But in this investigation, it is reported that by constructing triangle free graph out of it merely studying four Hamiltonian circuits, the process can be completed very easily in a very short span of time. It can be claimed that the maximal triangle free graph has played an important role in finding out the solution of traveling salesman problem, which is a new direction of traveling salesman problem.

Table-1

\begin{tabular}{|c|c|c|c|c|c|c|c|c|}
\hline & $1 \leq k \leq m-1$ & $1 \leq i \leq 2 m-1$ & $1 \leq j \leq 2 m-3$ & $1 \leq l \leq 2 m-5$ & $1 \leq p \leq 2 m-7$ & $\ldots \ldots$. & $\ldots \ldots$ & $\ldots \ldots$ \\
\hline$m=2$ & $v_{1} v_{2 k+2}$ & $v_{i} v_{i+4}$ & & & & $\ldots$ & $\ldots$ & $\ldots$ \\
\hline$m=3$ & $v_{1} v_{2 k+2}$ & $v_{i} v_{i+4}$ & $v_{j} v_{j+6}$ & & & $\ldots$ & $\ldots$ & $\ldots$ \\
\hline$m=4$ & $v_{1} v_{2 k+2}$ & $v_{i} v_{i+4}$ & $v_{j} v_{j+6}$ & $v_{l} v_{l+8}$ & & $\ldots$ & $\ldots$ & \\
\hline$m=5$ & $v_{1} v_{2 k+2}$ & $v_{i} v_{i+4}$ & $v_{j} v_{j+6}$ & $v_{l} v_{l+8}$ & $v_{p} v_{p+10}$ & $\ldots$ & $\ldots$ & . \\
\hline$\cdots$ & $\cdots$ & $\cdots$ & $\cdots$ & $\cdots$ & $\cdots$ & $\cdots$ & $\cdots$ & . \\
\hline$\ldots$ & $\ldots$ & $\ldots$ & $\ldots$ & $\ldots$ & $\ldots$ & $\ldots$ & $\ldots$ & $\cdots$ \\
\hline
\end{tabular}


Table-2

\begin{tabular}{|c|c|c|c|c|c|c|c|c|}
\hline & & $1 \leq p \leq m-1$ & $1 \leq i \leq m-1$ & $1 \leq j \leq m-1$ & $1 \leq k \leq m-2$ & $1 \leq l \leq m-3$ & $1 \leq q \leq m-4$ & $\ldots$ \\
\hline$m=2$ & $v_{2 m+1} v_{2 m+3}$ & & $v_{1} v_{i+2}, v_{1} v_{i+5}$ & $v_{2} v_{j+3}$ & & & & $\cdots$ \\
\hline$m=3$ & $v_{2 m+1} v_{2 m+3}$ & $v_{m+3} v_{p+7}$ & $v_{1} v_{i+2}, v_{1} v_{i+6}$ & $v_{2} v_{j+3}$ & $v_{3} v_{k+4}$ & & & $\cdots$ \\
\hline$m=4$ & $v_{2 m+1} v_{2 m+3}$ & $v_{m+3} v_{p+8}$ & $v_{1} v_{i+2}, v_{1} v_{i+7}$ & $v_{2} v_{j+3}$ & $v_{3} v_{k+4}$ & $v_{4} v_{l+5}$ & & $\cdots$ \\
\hline$m=5$ & $v_{2 m+1} v_{2 m+3}$ & $v_{m+3} v_{p+9}$ & $v_{1} v_{i+2}, v_{1} v_{i+8}$ & $v_{2} v_{j+3}$ & $v_{3} v_{k+4}$ & $v_{4} v_{l+5}$ & $v_{5} v_{q+6}$ & $\cdots$ \\
\hline$\ldots \ldots$ & $\ldots \ldots \ldots \ldots$ & $\ldots \ldots \ldots \ldots$ & …........... & $\cdots \cdots$ & ........... & $\ldots \ldots$ & $\ldots \ldots$ & $\ldots$ \\
\hline .......... & $\ldots \ldots \ldots \ldots \ldots$ & $\ldots \ldots \ldots \ldots$ & $\ldots \ldots \ldots \ldots$ & $\ldots \ldots \ldots$ & ............ & $\ldots \ldots \ldots$ & $\ldots \ldots$ & $\ldots$ \\
\hline
\end{tabular}

Table-3

\begin{tabular}{|c|c|c|c|c|c|c|c|c|c|c|c|}
\hline & & $\begin{array}{c}1 \leq i \leq \\
m-2\end{array}$ & $\begin{array}{c}1 \leq j \leq \\
m-2\end{array}$ & $\begin{array}{c}1 \leq k \leq \\
m-3\end{array}$ & $\begin{array}{c}1 \leq l \leq \\
m-4\end{array}$ & & & $\begin{array}{c}1 \leq p \leq \\
m-1\end{array}$ & $\begin{array}{c}1 \leq q \leq \\
m-2\end{array}$ & & \\
\hline$m=2$ & $\begin{array}{c}v_{1} v_{2,} v_{3} v_{4} \\
v_{1} v_{2 m+3} \\
v_{2 m+1} v_{2 m+2}\end{array}$ & & & & & $\cdots$ & & & & $\cdots$ & $\ldots$ \\
\hline$m=3$ & $\begin{array}{c}v_{1} v_{2}, v_{3} v_{4} \\
v_{1} v_{2 m+3} \\
v_{2 m+1} v_{2 m+2}\end{array}$ & $\begin{array}{c}v_{1} v_{i+3} \\
v_{1} v_{m+3+i} \\
v_{2} v_{i+4}\end{array}$ & & & & $\cdots$ & $v_{m} v_{m+2}$ & $v_{m+3} v_{m+4+p}$ & & $\ldots$ & $\ldots$ \\
\hline$m=4$ & $\begin{array}{c}v_{1} v_{2}, v_{3} v_{4} \\
v_{1} v_{2 m+3} \\
v_{2 m+1} v_{2 m+2}\end{array}$ & $\begin{array}{c}v_{1} v_{i+3} \\
v_{1} v_{m+3+i} \\
v_{2} v_{i+4}\end{array}$ & $v_{3} v_{j+4}$ & & & $\cdots$ & $v_{m} v_{m+2}$ & $v_{m+3} v_{m+4+p}$ & $v_{m+4} v_{m+5+q}$ & $\ldots$ & $\ldots$ \\
\hline$m=5$ & $\begin{array}{c}v_{1} v_{2}, v_{3} v_{4} \\
v_{1} v_{2 m+3} \\
v_{2 m+1} v_{2 m+2}\end{array}$ & $\begin{array}{c}v_{1} v_{i+3} \\
v_{1} v_{m+3+i} \\
v_{2} v_{i+4}\end{array}$ & $v_{3} v_{j+4}$ & $v_{4} v_{k+5}$ & & $\cdots$ & $v_{m} v_{m+2}$ & $v_{m+3} v_{m+4+p}$ & $v_{m+4} v_{m+5+q}$ & & \\
\hline$m=6$ & $\begin{array}{c}v_{1} v_{2}, v_{3} v_{4} \\
v_{1} v_{2 m+3} \\
v_{2 m+1} v_{2 m+2}\end{array}$ & $\begin{array}{c}v_{1} v_{i+3} \\
v_{1} v_{m+3+i} \\
v_{2} v_{i+4}\end{array}$ & $v_{3} v_{j+4}$ & $v_{4} v_{k+5}$ & $v_{5} v_{l+6}$ & $\cdots$ & $v_{m} v_{m+2}$ & $v_{m+3} v_{m+4+p}$ & $v_{m+4} v_{m+5+q}$ & $\ldots$ & $\ldots$ \\
\hline$\ldots \ldots$. & ............ & $\ldots \ldots \ldots$ & $\ldots \ldots \ldots$ & ........... & ............ & $\ldots$ & $\ldots \ldots \ldots$ & ............. & ............. & $\ldots$ & $\ldots$ \\
\hline$\ldots \ldots \ldots$ & .......... & ............. & $\ldots \ldots \ldots \ldots$ & $\ldots \ldots$ & .......... & $\ldots$ & $\ldots \ldots \ldots$ & ........... & $\ldots \ldots \ldots \ldots$ & $\ldots$ & $\ldots$ \\
\hline
\end{tabular}

Table-4

\begin{tabular}{|l|l|l|l|l|l|l|l|}
\hline & A & B & C & D & E & F & G \\
\hline A & $\infty$ & 4 & 60 & 33 & 30 & 42 & 7 \\
\hline B & 4 & $\infty$ & 9 & 38 & 20 & 27 & 36 \\
\hline C & 60 & 9 & $\infty$ & 21 & 47 & 19 & 23 \\
\hline D & 33 & 38 & 21 & $\infty$ & 18 & 56 & 13 \\
\hline E & 30 & 20 & 47 & 18 & $\infty$ & 11 & 51 \\
\hline F & 42 & 27 & 19 & 56 & 11 & $\infty$ & 24 \\
\hline G & 7 & 36 & 23 & 13 & 51 & 24 & $\infty$ \\
\hline
\end{tabular}

Table-5

\begin{tabular}{|l|l|l|l|l|l|l|l|}
\hline & A & B & C & D & E & F & G \\
\hline A & $\infty$ & 65 & 39 & 5 & 19 & 40 & 43 \\
\hline B & 65 & $\infty$ & 47 & 37 & 21 & 11 & 7 \\
\hline C & 39 & 47 & $\infty$ & 54 & 17 & 24 & 13 \\
\hline D & 5 & 37 & 54 & $\infty$ & 60 & 8 & 29 \\
\hline E & 19 & 21 & 17 & 60 & $\infty$ & 51 & 31 \\
\hline F & 40 & 11 & 24 & 8 & 51 & $\infty$ & 63 \\
\hline G & 43 & 7 & 13 & 29 & 31 & 63 & $\infty$ \\
\hline
\end{tabular}

Table-6

\begin{tabular}{|l|l|l|l|l|l|l|l|}
\hline & A & B & C & D & E & F & G \\
\hline A & $\infty$ & 31 & 51 & 15 & 2 & 33 & 29 \\
\hline B & 31 & $\infty$ & 47 & 42 & 5 & 19 & 7 \\
\hline C & 51 & 47 & $\infty$ & 30 & 23 & 10 & 8 \\
\hline D & 15 & 42 & 30 & $\infty$ & 40 & 12 & 26 \\
\hline E & 2 & 5 & 23 & 40 & $\infty$ & 28 & 38 \\
\hline F & 33 & 19 & 10 & 12 & 28 & $\infty$ & 35 \\
\hline G & 29 & 7 & 8 & 26 & 38 & 35 & $\infty$ \\
\hline
\end{tabular}




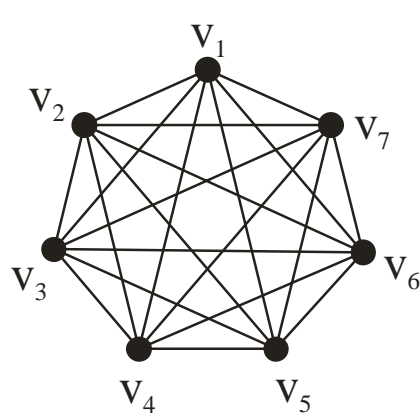

Figure-1

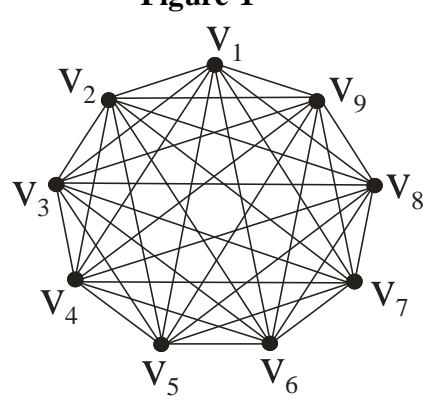

Figure-5

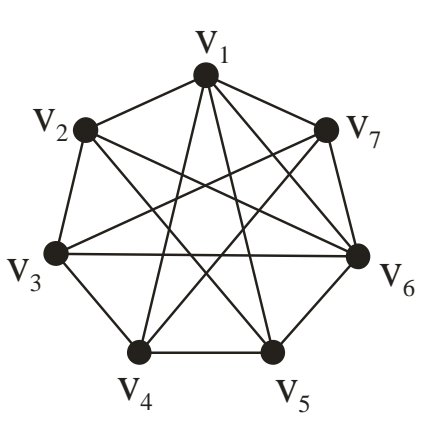

Figure-2

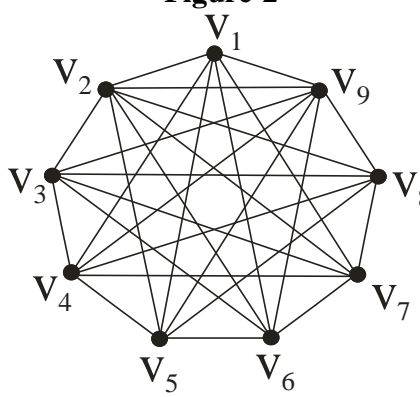

Figure-6

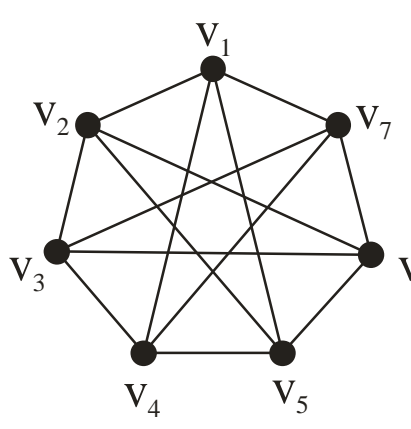

Figure-3

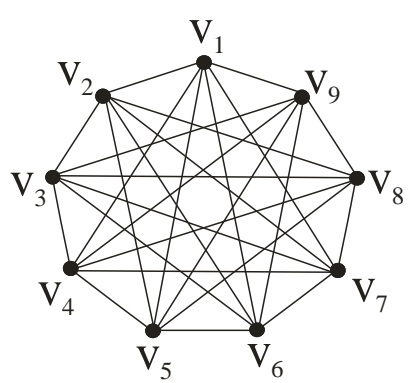

Figure-7

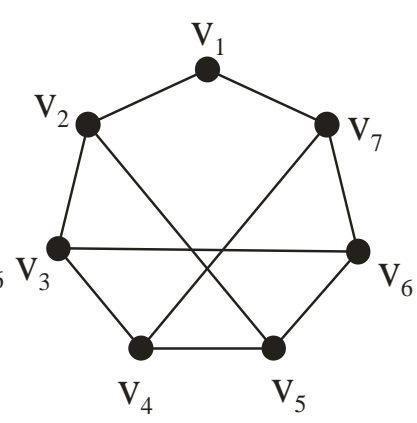

Figure-4

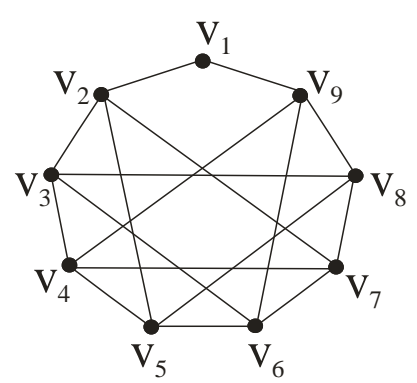

Figure-8

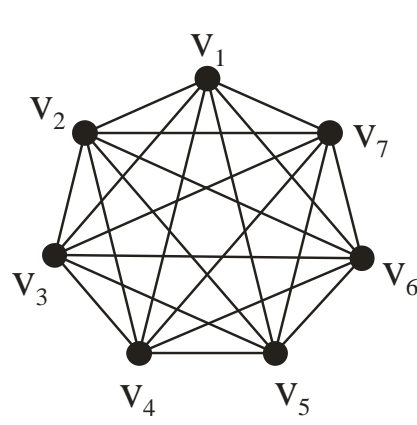

Figure-9

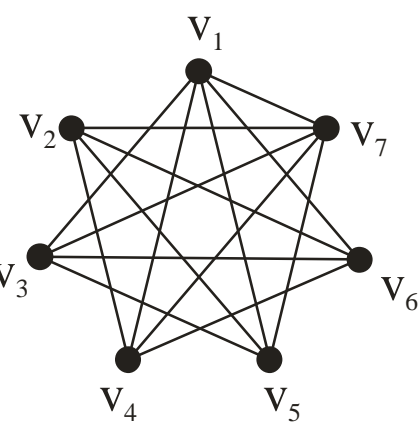

Figure-10

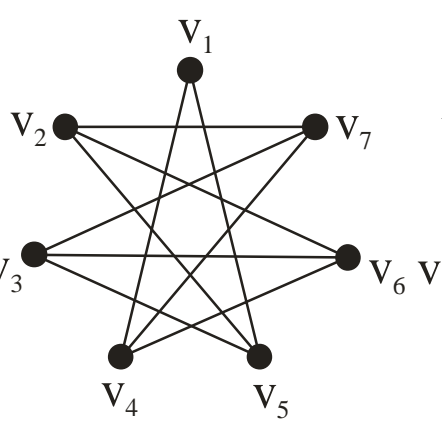

Figure-11

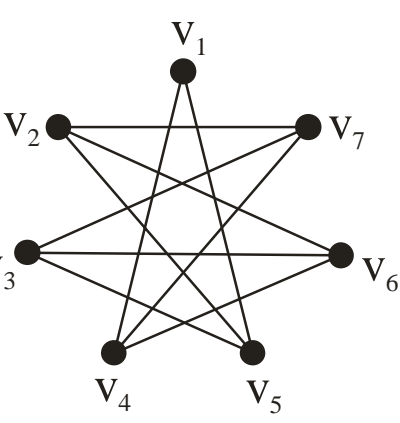

Figure-12

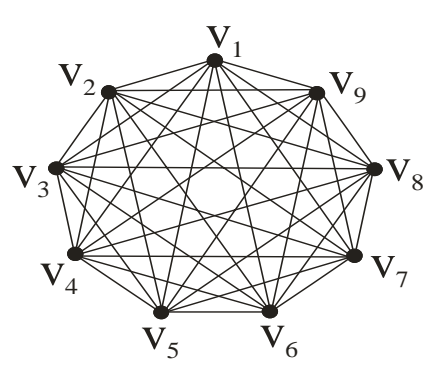

Figure-13

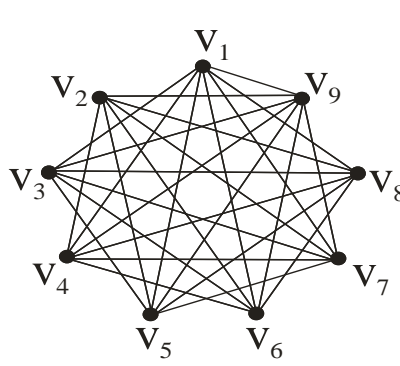

Figure-14

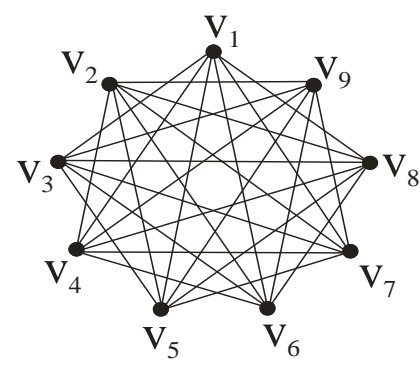

Figure-15

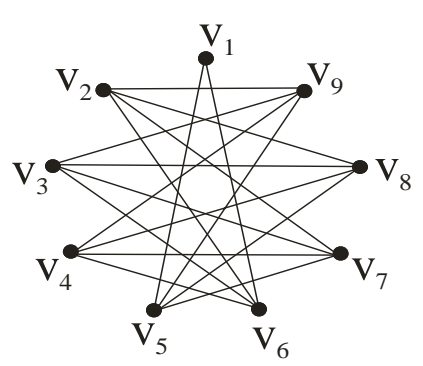

Figure-16

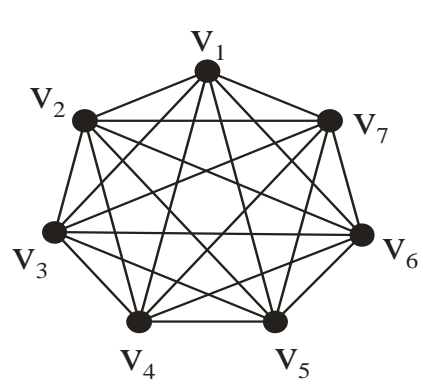

Figure-17

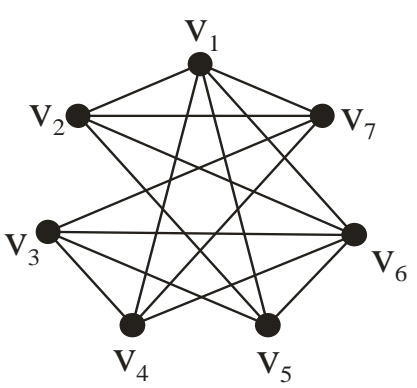

Figure-18

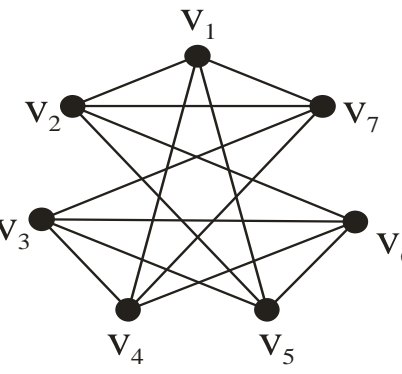

Figure-19

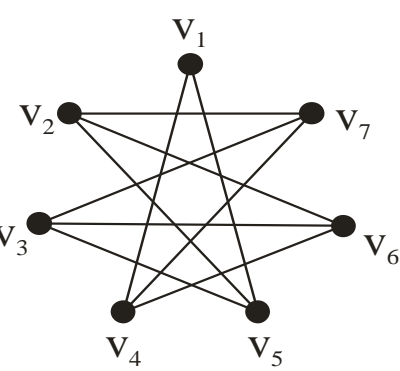

Figure-20 


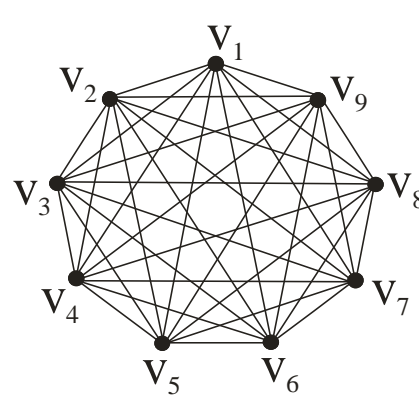

Figure-21

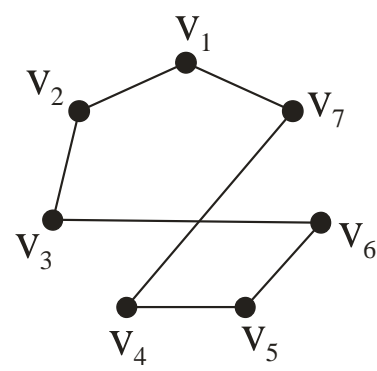

Figure-25a

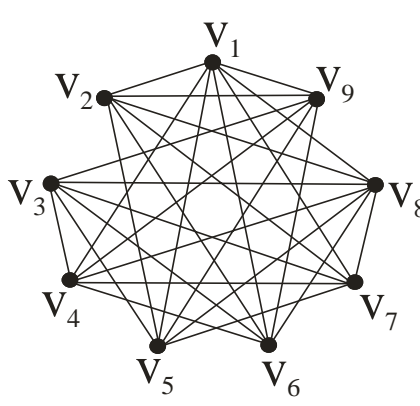

Figure-22

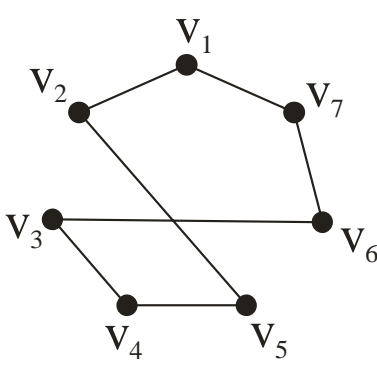

Figure-25b

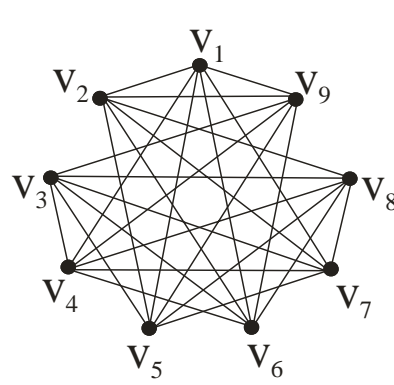

Figure-23

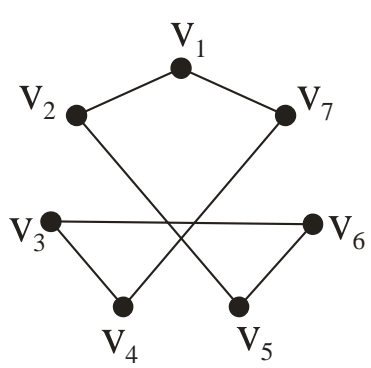

Figure-25c

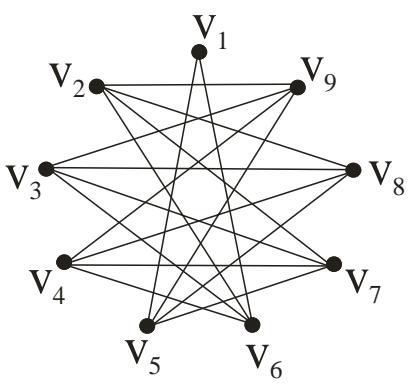

Figure-24

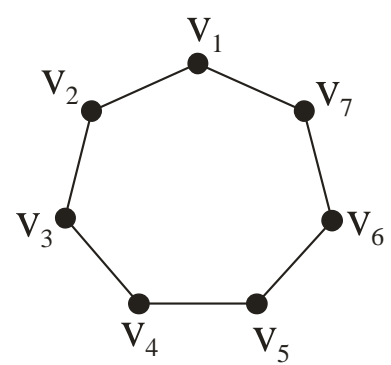

Figure-25d

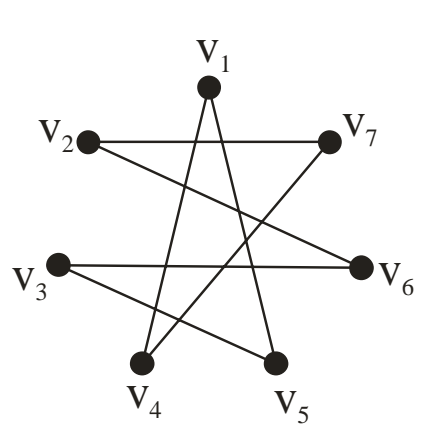

Figure-25e

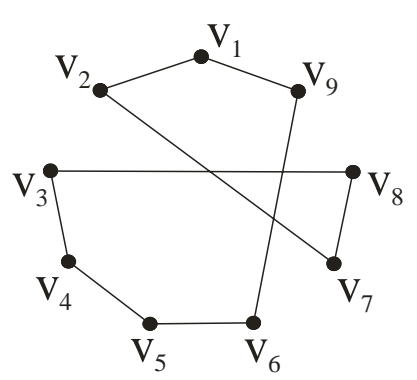

Figure-26a

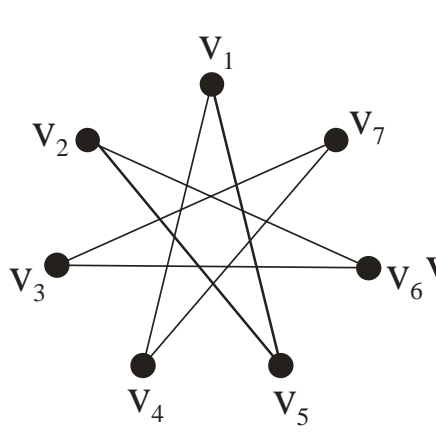

Figure-25f

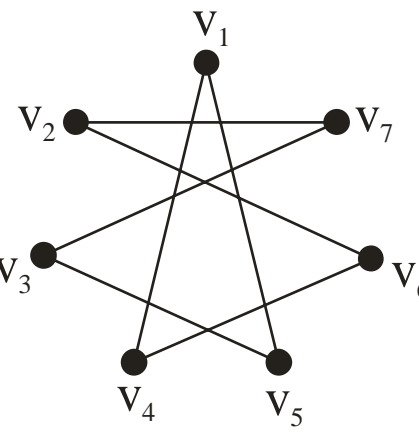

Figure-25g

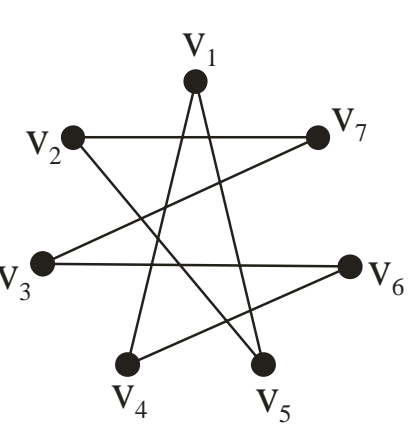

Figure-25h

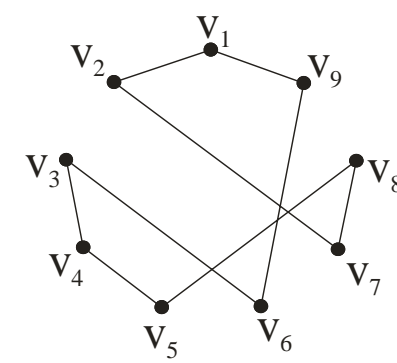

Figure-26b

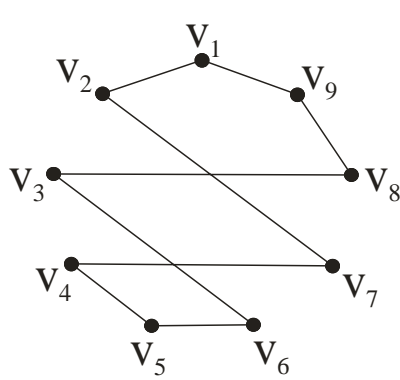

Figure-26c

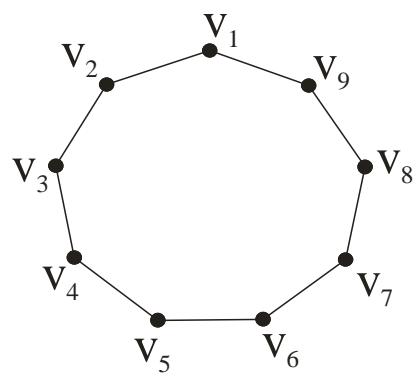

Figure-26d 


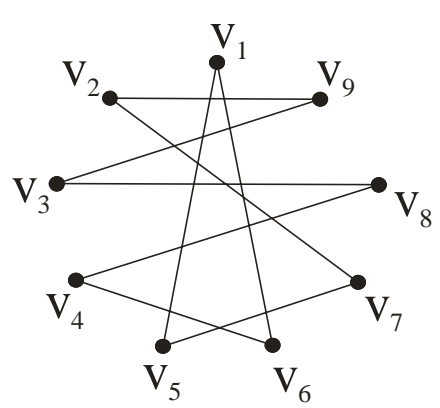

Figure-26e

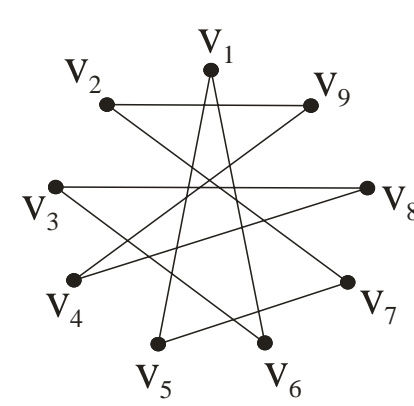

Figure-26f

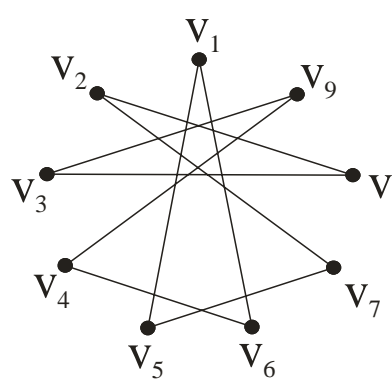

Figure-26g

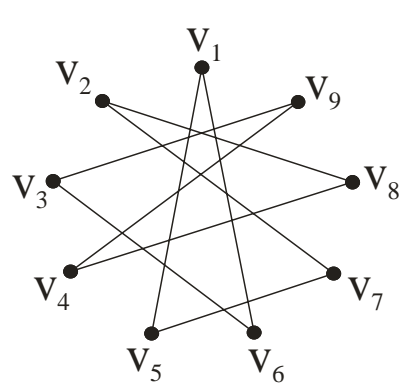

Figure-26h

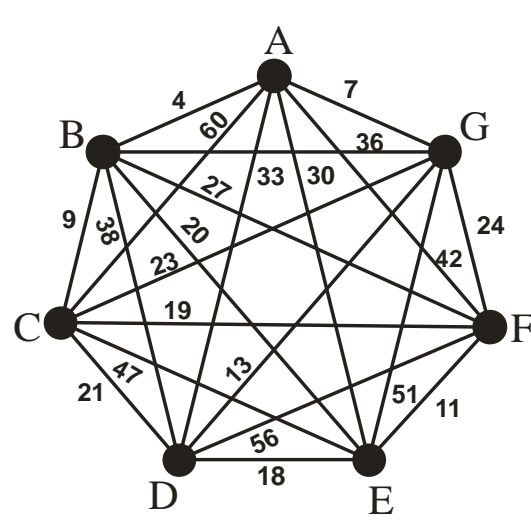

Figure-27

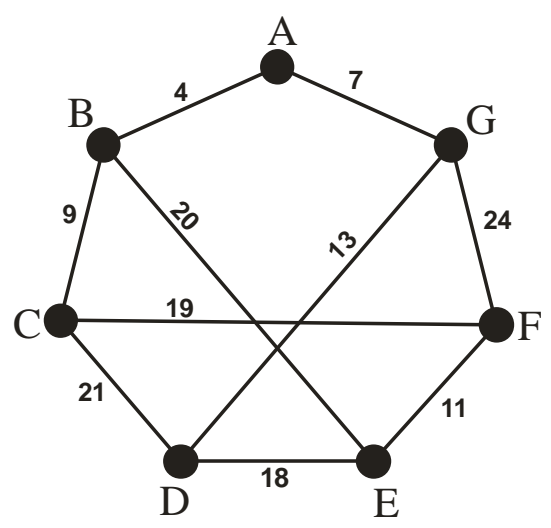

Figure-30

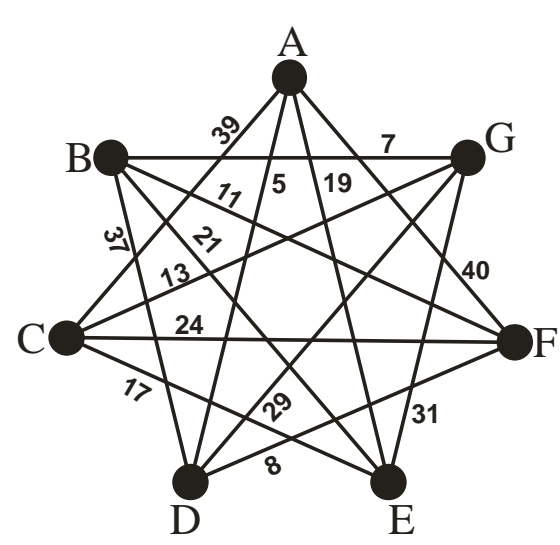

Figure-33

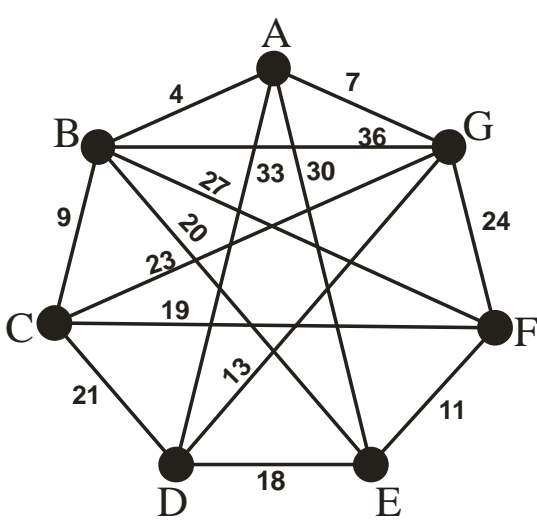

Figure-28

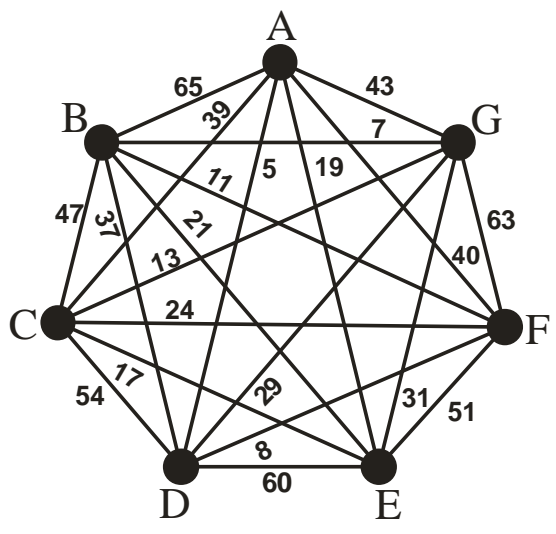

Figure-31

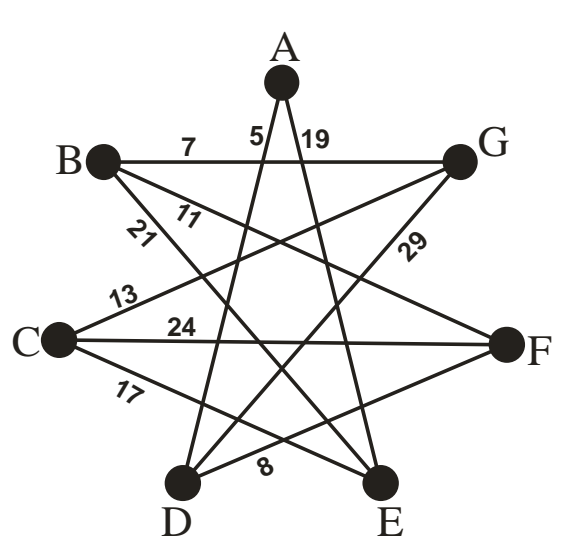

Figure-34

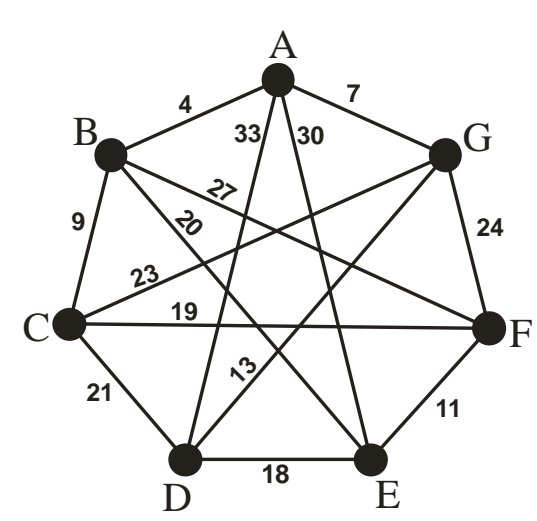

Figure-29

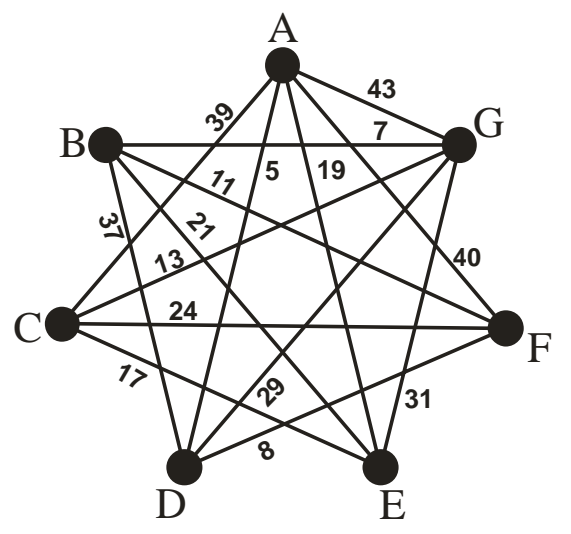

Figure-32

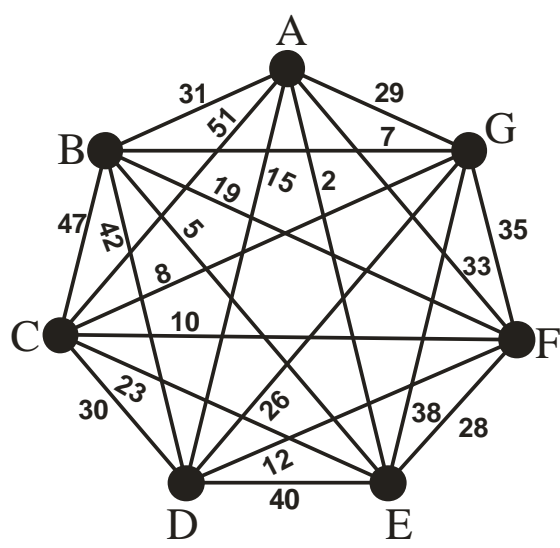

Figure-35 


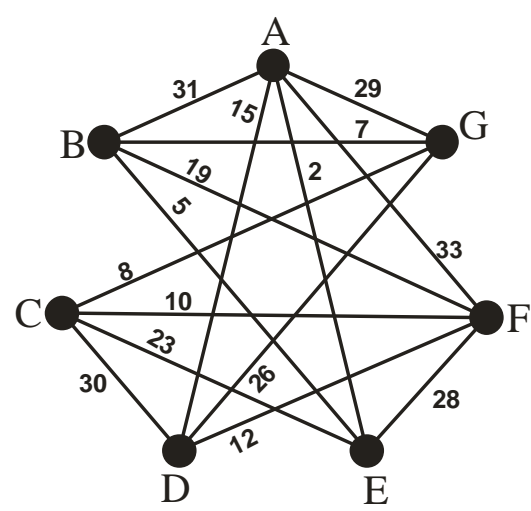

Figure-36

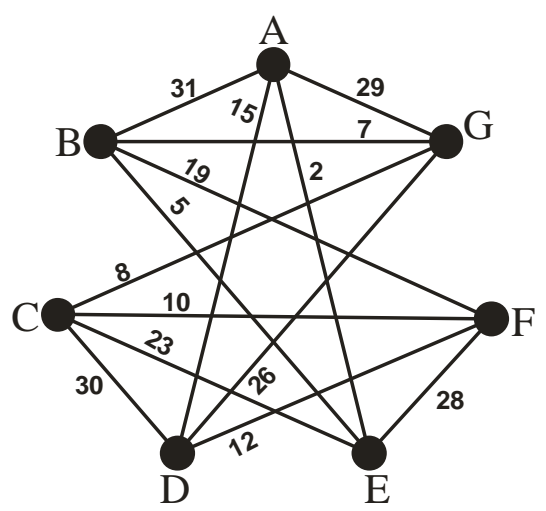

Figure-37

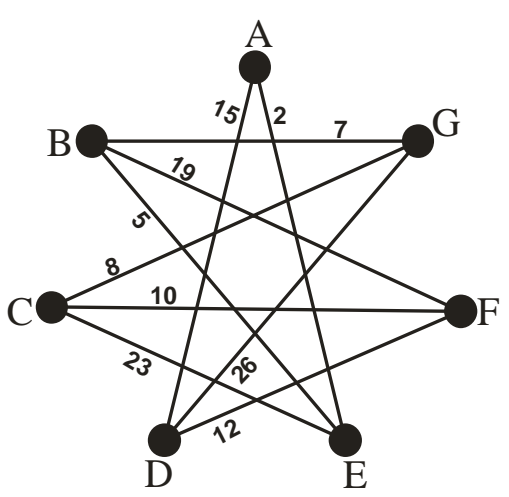

Figure-38

\section{REFERENCES}

[1] Woodall, D., 1973, "The Binding number of a graph and its Anderson number", J. Combin, Theory B 15, p.p. $225-255$.

[2] Shi, R., 1985, "The binding number of a graph and its triangle", Acta Math. Appl. Sinica (English series) 2, pp. $79-86$

[3] Goddard, W., Kleitman, D.J., 1993, "A note on maximal triangle free graphs", Journal in graph theory, Vol. 17, issue 5, pp. $629-631$.

[4] Furedi, Z., Reimer, D., A'kos Seress, 1991, "Hajnal's triangle-free game and extremal graph problems", Congressus Numerantium 82, p.p. 123 - 128.

[5] Briegmann, D., Komusiewiccz, C., Moser, H., 2009, "On Generating Triangle Free Graph", Proc. AGT.

[6] Brandt, S., Brinkmann, G. and Harmuth, T., (2000), "The Generator of Maximal Triangle Free Graph”, Graph \& combinatorics 16: 149-157.

[7] Kalita, B., 2005, "Some Investigation on Graph Theory, $\mathrm{PhD}$ thesis".Finance India. Vol. XIX, NO-4DEC (P.P. 1430-1438).
[8] Kalita, B., 2003, "Application of three edges extension of planar (TEEP) graph for the Solution of traveling salesman problem" Proc. $48^{\text {th }}$ ISTAM conference.

[9] Kalita, B., 2003, "Non-isomorphic Hamiltonian SubGraphs of complete graph and their application" Proc. Indian Sc. Congress, Ahmedabad.

[10] Kalita, B., 2006, "Sub Graph of Complete Graph" Proceeding, International Conference on Foundation of computer Science. Lasvegas, U.S.A. p.p. 71-77.

[11] Dutta. Anupam, Kalita. B., Baruah. H.K., 2010, "Regular sub-graph of complete graph" International Journal of Applied Engineering Research. Vol.5. No-8, p.p. 13151323.

[12] Dutta. A, Kalita. B, Baruah. H.K., (2010), "Regular Planar Sub-Graphs of Complete Graph and Their Application”, IJAER, Vol-5 no-3, p.p. 377-386.

[13] Choudhury, J.K., Kalita, B., 2011, "An Algorithm for TSP" Bulletin of Pure and Applied Sciences, Vol 30 E (Math \&Stat.) Issue (No.1), p.p.111-118.

[14] Choudhury, J.K., Dutta, A, Kalita,B., 2012, "Graph Factorization and Its Application", International Journals of Multidisciplinary Research Academy, Vol 2, Issue3, p.p. 208-220. 